\title{
Absence of weak localization in two-dimensional disordered Frenkel lattices
}

\author{
A. Rodríguez ${ }^{1}$, M. A. Martín-Delgado 2 , J. Rodriguez-Laguna ${ }^{2}$, G. Sierra ${ }^{3}$, V. A. \\ Malyshev $^{4}$, F. Domínguez-Adame ${ }^{5}$, and J. P. Lemaistre ${ }^{6}$ \\ ${ }^{1}$ GISC, Departamento de Matemática Aplicada y Estadística, Universidad Politécnica, E-28040 \\ Madrid, Spain \\ ${ }^{2}$ Departamento de Física Teórica I, Universidad Complutense, E-28040 Madrid, Spain \\ ${ }^{3}$ Instituto de Matemáticas y Física Fundamental, CSIC, Madrid, Spain \\ ${ }^{4}$ National Research Center "Vavilov State Optical Institute", 199034 Saint-Petersburg, Russia \\ ${ }^{5}$ GISC, Departamento de Física de Materiales, Universidad Complutense, E-28040 Madrid, Spain \\ ${ }^{6}$ Laboratoire des Milieux Désordonnés et Hétérogènes, Université P. et M. Curie, CNRS \\ UMR7603, Paris, France
}

(December 10, 2013)

\begin{abstract}
The standard one-parameter scaling theory predicts that all eigenstates in two-dimensional random lattices are weakly localized. We show that this claim fails in two-dimensional dipolar Frenkel exciton systems. The linear energy dispersion at the top of the exciton band, originating from the longrange inter-site coupling of dipolar nature, yields the same size-scaling law for the level spacing and the effective disorder seen by the exciton. This finally results in the delocalization of those eigenstates in the thermodynamic limit. Large scale numerical simulations allow us to perform a detailed multifractal analysis and to elucidate the nature of the excitonic eigenstates.
\end{abstract}


Keywords: Frenkel excitons, Anderson localization, disordered solids

Typeset using REVTEX 
One of the most attractive problems in condensed matter physics is the localization of quasi-particles (electrons, phonons, excitons) in disordered matter. The existence or absence of the localization-delocalization transition has been found to strongly depend on the system dimensionality. The one-parameter scaling theory of localization [1] states that any nonzero disorder causes exponential localization of all eigenstates in one-dimensional (1D) and twodimensional (2D) systems, regardless their energies, while in three-dimensional (3D) systems only a rather strong disorder causes the state localization. It is to be noted that there exist several exceptions to this rule. In this regard, anomalously weak localization is known to occur at the band center in 1D [2] and 2D [2,3] systems with off-diagonal disorder. Moreover, correlations in disorder may cause delocalization of states in $1 \mathrm{D}$ systems [4] even in the presence of strong disorder. The long-range inter-site coupling also may act as a driving force for delocalization [0,8] in any dimension. We have recently shown the absence of localization in a 1D Hamiltonian with a special type of long-range intersite interaction, resulting in a specific, non-parabolic quasi-particle energy dispersion. Remarkably, the delocalized states belong to one tail of the band [9].

In this work we report further progress along the lines mentioned above and demonstrate that extended states may occur in 2D disordered Frenkel systems, where the exciton eigenenergies of the ordered system scale linearly with the wavenumber at the top of the exciton band. In such a case, the level spacing decreases on increasing the system size in the same manner that the strength of effective disorder seen by the exciton [see Eq. (4) below]. Therefore, if the disorder is of perturbative magnitude for a given lattice size, it will remain perturbative on increasing the size, consequently allowing delocalized eigenstates.

We consider a Frenkel exciton Hamiltonian on a regular $\mathcal{N}=N \times N$ lattice with diagonal disorder:

$$
\mathcal{H}=\sum_{\mathbf{n}} \epsilon_{\mathbf{n}}|\mathbf{n}\rangle\left\langle\mathbf{n}\left|+\sum_{\mathbf{m n}} J_{\mathbf{n m}}\right| \mathbf{n}\right\rangle\langle\mathbf{m}|
$$

Here, $|\mathbf{n}\rangle$ is the state vector of the $\mathbf{n}$-th excited molecule with energy $\epsilon_{\mathbf{n}}$ and $\mathbf{n}=\left(n_{x}, n_{y}\right)$, $n_{x}, n_{y}$ being integers $\left(-N / 2 \leq n_{x}, n_{y}<N / 2\right.$, with $N$ even). The intersite dipole-dipole 
interaction is taken in the form $J_{\mathbf{n m}}=J /|\mathbf{n}-\mathbf{m}|^{3}$, where $J>0$ is the coupling between nearest-neighbor $(\mathrm{NN})$ molecules in the lattice (hereafter we assume that transition dipole moments of molecules are perpendicular to the plane of the system and that their magnitudes are the same). The joint distribution function of a realization of disorder is the product of box functions of width $\Delta$ centered around zero. The quantity $\Delta / J$ is referred to as degree of disorder.

In the excitonic representation, assuming periodic boundary conditions, the Frenkel Hamiltonian (1) takes the form

$$
\mathcal{H}=\sum_{\mathbf{K}} E_{\mathbf{K}}|\mathbf{K}\rangle\left\langle\mathbf{K}\left|+\sum_{\mathbf{K K}^{\prime}}(\delta \mathcal{H})_{\mathbf{K K}^{\prime}}\right| \mathbf{K}\right\rangle\left\langle\mathbf{K}^{\prime}\right|
$$

where $\mathbf{K}=(2 \pi / N)\left(k_{x}, k_{y}\right)$ runs over the first Brillouin zone, $k_{x}, k_{y}$ being integers ranging within the interval $-N / 2 \leq k_{x}, k_{y}<N / 2$. Here $E_{\mathbf{K}}$ is the unperturbed exciton eigenenergy

$$
E_{\mathbf{K}}=J \sum_{\mathbf{n} \neq \mathbf{0}} \frac{1}{|\mathbf{n}|^{\mathbf{3}}} e^{i \mathbf{K} \cdot \mathbf{n}}
$$

and $(\delta \mathcal{H})_{\mathbf{K K}^{\prime}}$ is the inter-mode coupling matrix

$$
(\delta \mathcal{H})_{\mathbf{K} \mathbf{K}^{\prime}}=\frac{1}{\mathcal{N}} \sum_{\mathbf{n}} \epsilon_{\mathbf{n}} e^{i\left(\mathbf{K}-\mathbf{K}^{\prime}\right) \cdot \mathbf{n}}
$$

Hereafter we keep long-range terms in (2b) due to their major role. It can be shown that near the extreme points of the band $\mathbf{K}=\mathbf{0}$ and $\mathbf{K}=\boldsymbol{\pi} \equiv(\pi, \pi)$ the exciton energy spectrum takes a linear and a parabolic form, respectively 10:

$$
\begin{aligned}
& E_{\mathbf{K}} \simeq 9.03 J-2 \pi J|\mathbf{K}|, \quad|\mathbf{K}| \ll 1, \\
& E_{\mathbf{K}} \simeq-2.65 J+0.4 J|\mathbf{K}-\boldsymbol{\pi}|^{2}, \quad|\mathbf{K}-\boldsymbol{\pi}| \ll 1 .
\end{aligned}
$$

From this it follows that the energy spacing close to the top of the exciton band behaves as $N^{-1}=\mathcal{N}^{-1 / 2}$, while in the vicinity of the bottom scales as $N^{-2}=\mathcal{N}^{-1}$.

Depending on the degree of disorder and the lattice size, the operator $\delta \mathcal{H}$ may couple the extended excitonic states $|\mathbf{K}\rangle$ to each other, thus resulting in their localization. Our task now is to calculate the typical fluctuation of this matrix in order to gain insight into the 
magnitude of the exciton inter-mode coupling. The corresponding magnitude of interest is $\sigma_{\mathbf{K K}^{\prime}}^{2}=\left\langle\left|(\delta \mathcal{H})_{\mathbf{K K}^{\prime}}\right|^{2}\right\rangle$, where the angular brackets indicate the average over the distribution $\prod_{\mathbf{n}} P\left(\epsilon_{\mathbf{n}}\right)$. After performing the average one gets

$$
\sigma_{\mathbf{K K}^{\prime}} \sim \sigma \equiv \frac{\Delta}{\sqrt{\mathcal{N}}}
$$

Here $\sigma$ is referred to as effective degree of disorder. As we can see, the typical magnitude of the inter-mode coupling scales as $\mathcal{N}^{-1 / 2}=N^{-1}$. The most remarkable fact is that $\sigma$ decreases on increasing $\mathcal{N}$ in the same manner as the level spacing close to the top of the exciton band. This finding has a dramatic effect on the localization properties of the states within this region. Indeed, consider for example a finite lattice of size $N \times N$ and the two first unperturbed states with $\mathbf{K}=\mathbf{0}$ and $\mathbf{K}^{\prime}=(2 \pi / N, 0)$. The energy difference between them, according to Eq. (3a), is $\delta E=4 \pi^{2} J / N$. Take now the degree of the disorder to be $\Delta \ll 4 \pi^{2} J$. Notice that this condition is not very restrictive since in actual systems the degree of disorder is expected not to exceed the exciton bandwith $(\simeq 11.68 J)$. Under this condition, the strength of the effective disorder $\sigma=\Delta / N$, governing the exciton state mixing and thereof localization, is of perturbative magnitude, namely $\sigma \ll \delta E$. What is most important, it will remain perturbative upon increasing the lattice size because both magnitudes scale similarly with $N$. Hence, these states will not be mixed by disorder and will remain extended over the entire lattice independent of its size. It is clear that the same conclusion can be drawn for all the states of the linear spectrum range except the degenerate ones. They are mixed by any small amount of disorder. However, since different sets of degenerate states are not coupled to each other due to the perturbative nature of the effective degree of disorder $(\sigma \ll \delta E)$, the above conclusion is also valid with regard to degenerate states.

Let us now turn to the parabolic range of the energy spectrum, where the level spacing decreases as $\mathcal{N}^{-1}=N^{-2}$ upon increasing the lattice size, i.e., faster than the effective degree of disorder $\sigma$ (the same behavior takes place for both edges of the band obtained within the NN approximation, namely taking $J_{\mathbf{n m}}=0$ when $|\mathbf{n}-\mathbf{m}|>1$ ). Now, even if one starts 
with a perturbative magnitude of $\Delta$ at a fixed lattice size (so that $\sigma \ll \delta E$ ), it becomes non-perturbative for larger sizes, resulting finally in localization of those eigenstates.

The results of numerical diagonalization of the Hamiltonian (1), by means of the Lanczos method [1], unambiguously confirm our qualitative arguments. To examine the character of the exciton eigenfunction (localized or extended) we have calculated the inverse participation ratio (IPR) of the uppermost exciton state, according to the standard definition IPR = $\sum_{\mathbf{n}}\left|\Psi_{\nu \mathbf{n}}\right|^{4}$, where the sum runs over lattice sites and it is assumed that the eigenfunction $\Psi_{\nu \mathbf{n}}$ of the $\nu$ th eigenstate is normalized to unity. On increasing the lateral size $N$, the IPR scales as $N^{-2}$ for delocalized states, spreading uniformly over a 2D system. On the contrary, localized states exhibit constant values for different $N$.

The IPR as a function of the lateral size $N$ is shown in Fig. 1 when the dipole-dipole interaction between all molecules is taken into account as well as within the NN approximation. The plots comprise the result of 20 averages over disorder realizations and $\Delta=J$ in all cases. The slope of the straight line, being equal to -1.91 when dipole-dipole interaction is taken into account, is close to the theoretical value -2 . This scaling suggests the fairly extended nature of the uppermost exciton state. Notice that there is no scaling of the IPR within the NN approximation, in perfect agreement with the well-known results stating that those exciton states are localized. Figure 2 shows an increase of the IPR at a threshold value $\simeq 13 \mathrm{~J}$, suggesting the occurrence of a smooth delocalization-localization transition.

A comprehensive way to characterize the spatial distribution of eigenfunctions is the computation of the singularity spectrum, as explained in, e.g., Ref. [12]. If we cover the system with $(M / L)^{2}$ boxes of size $L^{2}$ (in units of the lattice spacing) and define the normalized $q$ th moments $\mu_{k}(q, \delta)=\mu_{k}^{q}(\delta) / \sum_{k^{\prime}} \mu_{k^{\prime}}^{q}(\delta)$ (where $\delta=L / M$ ) of the probability distribution $\mu_{k}(\delta)=\sum_{n \in \text { box } k}\left|\Psi_{n}\right|^{2}$ of finding an exciton in the $k$ th box, we may calculate the Lipschitz-Hölder exponents

$$
\alpha(q)=\lim _{\delta \rightarrow 0} \sum_{k} \mu_{k}(q, \delta) \ln \mu_{k}(1, \delta) / \ln \delta
$$

which take into account the scaling of the content of each box with the box size, as well as 
the corresponding value of the singularity spectrum

$$
f(q)=\lim _{\delta \rightarrow 0} \sum_{k} \mu_{k}(q, \delta) \ln \mu_{k}(q, \delta) / \ln \delta
$$

The invariance of the singularity spectrum with the system size for a given value of disorder is usually taken [12 as a proof of the occurrence of the Anderson transition. Nevertheless strong fluctuations of the eigenstates of the system near the transition makes it difficult to calculate the $f(\alpha)$ curve for the threshold value of $\Delta$. We have calculated the singularity spectrum for values of the degree of disorder below the threshold. Figure 3 shows the broadening of the $f(\alpha)$ curve with increasing disorder, indicating that the excitonic eigenfunctions at the top of the band becomes progressively more and more localized. This result suggests again the occurrence of a smooth delocalization-localization transition, as in Fig. Z.

In summary, we have shown that the statement of the one-parameter scaling theory [1] about the weak localization in two dimensions, i.e., that any amount of disorder results in localization of all eigenstates, fails near the top of the exciton band where the quasiparticle spectrum scales linearly with the wavenumber $|\mathbf{K}|$. The states lying at such energy range are delocalized at moderate strength of disorder and undergo the continuous Anderson transition as the disorder degree increases. In our opinion, the failure of the one-parameter scaling theory for the conditions considered in the present work is due to the fact that this theory deals only with the size scaling of the energy spacing but pays no attention to the subsequent renormalization of the disorder (4). As it follows from our treatment, the latter effect plays a major role in localization phenomena, violating the one-parameter scaling and thus leading to the impossibility to match our results by this theory.

The authors thank E. Maciá, A. Sánchez, E. Diez, R. Römer and M. Hilke for discussions. F. D-A. and A. R. were supported by DGI-MCyT (Project MAT2000-0734). V. A. M. acknowledges support from INTAS (Project No. 97-10434). 


\section{REFERENCES}

[1] E. Abrahams, P. W. Anderson, D. C. Licciardello, and V. Ramakrishnan, Phys. Rev. Lett. 42, 673 (1979).

[2] M. Inui, S. A. Trugman, and E. Abrahams, Phys. Rev. B 49, 3190 (1994).

[3] A. Eilmes, R. A. Römer, and M. Schreiber, Eur. Phys. J. B 1, 29 (1998); phys. stat. sol. (b) 205, 229 (1998).

[4] J. C. Flores, J. Phys.: Condens. Matter 1, 8471 (1989).

[5] D. H. Dunlap, H.-L. Wu, and P. Phillips, Phys. Rev. Lett. 65, 88 (1990).

[6] V. Bellani, E. Diez, R. Hey, L. Toni, L. Tarricone, G. B. Parravicini, F. DomínguezAdame, and R. Gómez-Alcalá, Phys. Rev. Lett. 82, 2159 (1999).

[7] D. E. Logan and P. G. Wolynes, Phys. Rev. B 29, 6560 (1984); ibid. 31, 2437 (1985); ibid. 36, 4135 (1987); J. Chem. Phys. 87, 7199 (1987).

[8] L. S. Levitov, Ann. Phys. (Leipzig) 8, 507 (1999).

[9] A. Rodríguez, V. A. Malyshev, and F. Domínguez-Adame, J. Phys. A: Math. Gen. 33 L161 (2000).

[10] P. L. Christiansen, Yu. B. Gaididei, M. Johansson, K. Ø. Rasmussen, V. K. Mezentsev, and J. Juul Rasmussen, Phys. Rev. B 57, 11303 (1998).

[11] G. H. Golub and C. F. Van Loan, Matrix Computations (The Johns Hopkins University Press, Maryland, 1996).

[12] K. H. Hoffmann and M. Schreiber (Eds.), Computational Physics (Springer, Berlin, 1996). 


\section{FIGURES}

FIG. 1. Lateral size scaling of the inverse participation ratio of the uppermost exciton eigenfunction at $\Delta=J$, obtained by averaging over 20 realizations of the disorder.

FIG. 2. IPR of the uppermost exciton eigenfunction as a function of the degree of disorder for a system of size $N \times N$ (shown in the plot). Results comprise 10 realizations of disorder.

FIG. 3. Singularity spectrum $f(\alpha)$ of the uppermost exciton eigenfunction for various degrees of disorder for a system of size $96 \times 96$. Results comprise 5 realizations of disorder. 
Figure 1

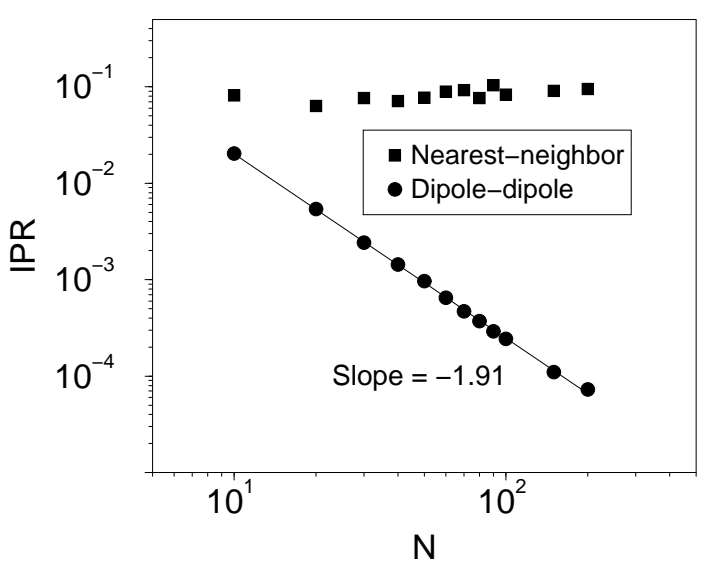

Figure 2

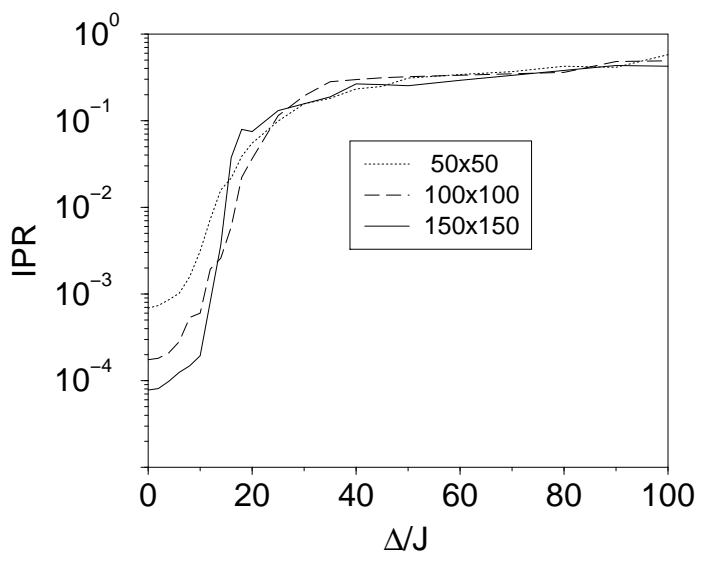

Figure 3

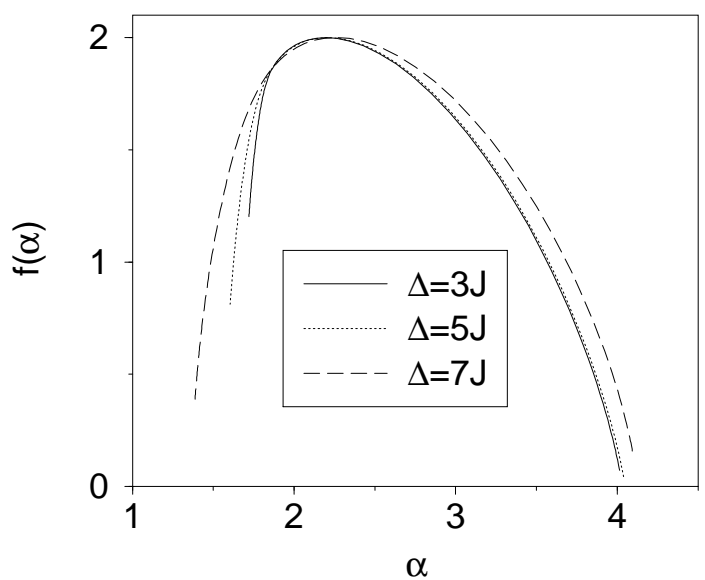

